Pacific Journal of Mathematics

ENDOMORPHISMS OF FUNCTION-SPACES WHICH LEAVE 


\title{
ENDOMORPHISMS OF FUNCTION-SPACES WHICH LEAVE STABLE ALL TRANSLATION-INVARIANT MANIFOLDS
}

\author{
R. E. EDWARDS
}

\section{The problem. Preliminary definitions}

0.1. Throughout $\S \S 0-5$ of this paper $X$ denotes a separated locally compact group with left Haar measure $d x$. Most of the time we shall consider a topological vector space $F$ of complex-valued functions, measures or distributions on $X$ which is invariant under left [right] translations $\tau_{a}\left[\rho_{a}\right](a \in X)$. By a left [right] invariant manifold in $F$ we shall mean a closed, left [right] translation invariant vector subspace of $F$. Our theorems result from an attempt to derive a general representation formula for those endomorphisms $T$ of $F$ which have the property of leaving stable left [right] invariant manifolds in $F$, i.e. which are such that $T(M) \subset M$ for each invariant manifold $M$ in $F$. This demand is tantamount to requiring that $T f \in M_{f}$ for each $f \in F$, where $M_{f}$ is the left [right] invariant manifold in $F$ generated by $F . \quad M_{f}$ is the intersection of all left [right] invariant manifolds in $F$ which contain $f$; and, if each translation operator $\tau_{a}\left[\rho_{a}\right]$ is a continuous endomorphism of $F, M_{f}$ is simply the closure in $F$ of the set of all finite linear combinations of translates $\tau_{a} f\left[\rho_{a} f\right]$ of $f$.

In most cases one may broaden the problem by demanding merely that $T f$ be the limit in some specified sense of finite linear combinations of $\tau_{a} f\left[\rho_{a} f\right]$, this sense being not necessarily derived from the initial topology of $F$. In fact, no topology may be involved which is related to $F$ in particular.

The type of representation theorem which appears in those cases which have been handled successfully asserts that $T f$ must take the form of a convolution $\mu * f[f * \mu]$, where $\mu$ is some measure or distribution on $X$ depending only upon $T$. Hidden within this conclusion are the facts that $T$ commutes with right [left] translations; and, in some cases at least, that $T$ is automatically continuous. On the other hand, there are cases where we have found it necessary to assume continuity of $T$ at the outset, so that the role of this hypothesis is not too clear.

I am indebted to a referee for pointing out to me a result due to P. Eymard [2], which asserts that, if $X$ is any Lie group, and if $T$ belongs to the von Neumann algebra of operators on $L^{2}(X)$ generated by left translations, then there exists a Schwartz distribution $\mu$ on $X$ such that $T f=\mu * f$ for each $f \in C_{c}^{\infty}(X)$. This result is closely related to Theorem 4.1 infra.

Received February 25, 1963. 
0.2 Finitely representable groups and representing sets. We shall say that the locally compact group $X$ is $F . R$. (short for finitely representable) if there exists a set $X^{\prime}=\{\xi\}$ of bounded, measurable, finite-dimensional representations $\xi$ of $X$ which is complete in the sense that if $\mu$ is a bounded Radon measure on $X$, and if

$$
\hat{\mu}(\xi) \equiv \int \xi(x) d \mu(x)
$$

vanishes for all $\xi \in X^{\prime}$, then $\mu=0$. Such a set $X^{\prime}$ is termed a representing set for $X$. We note that each $\xi \in X^{\prime}$ is necessarily continuous and equivalent to a unitary representation of $X$ (see [8], pp. 66-67, 70).

We remark in passing that if the bounded measure $\mu$ is a function $f$ (i.e. has density $f$ with respect to $d x$ ), which is necessarily integrable, then we shall write $\hat{f}(\xi)$ in place of $\hat{\mu}(\xi)$. Thus $\hat{f}(\xi)$ is written for $\int \xi(x) f(x) d x$ whenever $f$ is an integrable function.

In all cases we shall write $V_{\xi}$ for the representation space of $\xi$, and $E_{\xi}$ for the endomorphism algebra of $V_{\xi}$. Thus for each $x \in X, \xi(x)$ is an invertible element of $E_{\xi}$.

Two important special cases are to be noted. First, if $X$ is abelian, one may take $X^{\prime}=\hat{X}$, the dual group of $X$, thereby identifying each bounded, continuous character of $X$ (an element of $\hat{X}$ ) with the onedimensional representation it defined. Secondly, if $X$ is compact, one may according to the Peter-Weyl theory take for $X^{\prime}$ a complete set of inequivalent, irreducible, continuous, unitary representations of $X$. Consequently, amongst the $F . R$. groups are to be found all abelian and all compact groups. By forming the tensor products of the corresponding representations, one sees that any finite product of $F . R$. groups is an $F . R$. group.

I do not know to what extent, if any, the subsequent results depend essentially on the hypothesis that $X$ be $F \cdot R$.

0.3 Various function spaces. We collect here a list of the principal function-spaces over $X$ which figure in our results.

First come the usual Lebesgue spaces $L^{p}=L^{p}(X)$ with $1 \leqq p \leqq \infty$ with their usual topologies and norms $\|\cdot\|_{p}$, formed relative to left Haar measure on $X$.

$M(X)$ denotes the space of all Radon measures on $X$. (For us the term "measure" will always mean "Radon measure".) The subspace of bounded measures is written $M_{b d}(X)$ and is normed in the usual way: $\|\mu\|=\int d|\mu|(x)$.

$C(X)$ is the space of all continuous functions on $X$; its "natural" topology is that of locally uniform convergence. On its subspace $C_{b d}(X)$ formed of the bounded, continuous functions, we introduce the usual 
"sup norm", $\|f\|=\operatorname{Sup}\{|f(x)|: x \in X\} . C_{b d}(X)$ contains $C_{0}(X)$, the space of functions in $C_{b d}(X)$ which tend to zero at infinity, which is normed as a subspace of $C_{b d}(X)$. Finally, $C_{c}(X)$ is the space of continuous functions with compact supports; its "natural" topology is as the inductive limit of the subspaces $C(X, K)=\{f \in C(X)$ : support $f \subset K\}, K$ ranging over all (or over a base for) the compact subsets of $X$, each $C(X, K)$ being endowed with the sup norm.

It is standard procedure to identify $M_{b d}$ with the dual of $C_{0}$, and with a subspace of the dual of $C_{b d} . M$ is the dual of $C_{c}$, and its associated weak topology $\sigma\left(M, C_{c}\right)$ is sometimes referred to as the "vague topology".

It is also customary to inject each locally integrable function $f$ into $M$, identifying $f$ with the measure $f d x$. This injects $L^{1}$ into $M_{b d}$.

A few words concerning the definition of $\tau_{a}$ and $\rho_{a}$ are in order. The natural definition of $\tau_{a}$ for functions is

$$
\tau_{a} f(x)=f\left(a^{-1} x\right),
$$

and for measures $\mu$ we then define $\tau_{a} \mu$ via the formula

$$
\int k d\left(\tau_{a} \mu\right)=\int\left(\tau_{a-1} k\right) d \mu \quad\left(k \in C_{c}\right) .
$$

The two definitions agree when we identify the function $f$ with the measure $f d x$. On turning to right translates, an assymetry creeps in because of our use of left Haar measure in the identification of functions with measures. For our purposes it seems overall simplest to define $\rho_{a} f$ for a function $f$ by

$$
\rho_{a} f(x)=\Delta(a) f\left(x a^{-1}\right),
$$

whilst $\rho_{a} \mu$ is defined when $\mu$ is a measure by the formula

$$
\int k d\left(\rho_{a} \mu\right)=\Delta(a) \int\left(\rho_{a-1} k\right) d \mu=\int k(x a) d \mu(x),
$$

where $\Delta(a)$ is the modular function of $X$. Thus $\Delta$ is defined by

$$
\int k(x a) d x=\Delta(a) \int k(x) d x
$$

for all $k \in C_{c} . \quad \Delta$ is a strictly positive, continuous character of $X$, and

$$
\int k\left(x^{-1}\right) d x=\int k(x) \Delta(x) d x
$$

Naturally, $\Delta \equiv 1$ if $X$ is abelian; this is also the case when $X$ is compact and when $X$ is a semisimple Lie group (see [8], p. 39).

With our definition of the $\rho_{a}$ one has the formulae 


$$
\alpha * \beta=\int\left(\tau_{x} \beta\right) d \alpha(x)=\int\left(\rho_{x} \alpha\right) d \beta(x)
$$

for (suitably restricted) measures $\alpha$ and $\beta$.

Notice also the formula

$$
\left\|\rho_{x} f\right\|_{L^{p}}=\Delta(x)^{1 / p^{\prime}}\|f\|_{L^{p}}
$$

for $1 \leqq p \leqq \infty$ and $1 / p+1 / p^{\prime}=1$.

Finally, if $X$ is a finite product of real lines or of circles, we shall use $\mathscr{D}=\mathscr{D}(X)=C_{c}^{\infty}(X)$, the space of indefinitely differentiable functions with compact supports. This is topologised as the inductive limit of the spaces $C^{\infty}(X, K)$, composed of the indefinitely differentiable functions with support contained in $K$ and endowed with the topology of uniform convergence of the function and each of its partial derivatives (of any order). The dual of $\mathscr{D}$ is $\mathscr{D}^{\prime}=\mathscr{D}^{\prime}(X)$, the space of distributions. $M(X)$ may be injected into $\mathscr{D}^{\prime}(X)$.

0.4 An algebraic lemma. Suppose that $V$ is a finite-dimensional vector space and that $E$ is its endomorphism algebra. Let $J$ be a linear map of $E$ into itself with the property that, for each $A \in E, J(A)$ belongs to the left [right] ideal in $E$ generated by $A$. Then there exists $C \in E$ such that $J(A)=C A[A C]$ for all $A \in E$.

Proof of the lemma. This is due to Dr Tekla Taylor. We give the proof for the "left-handed" case.

Let $n$ be the dimension of $V$. Introducing a base for $V$ we may refer to endomorphisms of $V$ via their $n \times n$ matrix representatives. In what follows $i, j, k, \alpha, \beta$ run over the natural numbers from 1 to $n$ inclusive, and the $(i, j)$ th entry in a matrix $A$ is denoted by $A(i, j)$.

Choose matrices $C_{i}$ and $C_{i j}$ so that $J\left(E_{i}\right)=C_{i} E_{i}$ and $J\left(E_{i j}\right)=C_{i j} E_{i j}$, where $E_{i j}(\alpha, \beta)=\delta_{i \alpha} \delta_{j \beta}$ and $E_{i}=\Sigma_{i} E_{i j}$. By additivity of $J$ one has

$$
C_{i} E_{i}=\Sigma_{j} C_{i j} E_{i j} \text {. }
$$

On evaluating the products this leads to the relations

$$
C_{i}(\alpha, i)=C_{i \beta}(\alpha, i),
$$

whence it follows after calculations that $J\left(E_{i j}\right)$ is also equal to $C_{i} E_{i j}$.

Next we observe that if the $C_{i}^{\prime}$ are defined by

$$
C_{i}^{\prime}(\alpha, \beta)=\delta_{i \beta} C_{i}(\alpha, i),
$$

then $C_{i}^{\prime} E_{i}=C_{i} E_{i}=J\left(E_{i}\right)$. The preceding argument shows that therefore $J\left(E_{i j}\right)=C_{i}^{\prime} E_{i j}$.

Moreover one may verify that $C_{k}^{\prime} E_{i j}=0$ if $k \neq i$.

Thus by linearity of $J$, if we put $C=\Sigma_{k} C_{k}^{\prime}$, one will have for 
arbitrary scalars $c_{i j}$ the formulae

$$
\begin{aligned}
J\left(\Sigma_{i j} c_{i j} E_{i j}\right) & =\Sigma_{i, j} c_{i j} C_{i}^{\prime} E_{i j}=\Sigma_{i} C_{i}^{\prime} \Sigma_{j} c_{i j} E_{i j}=\Sigma_{i} C \Sigma_{j} c_{i j} E_{i j} \\
& =C\left(\Sigma_{i, j} c_{i j} E_{i j}\right) .
\end{aligned}
$$

This proves the lemma, since the $E_{i j}$ form a base for the space of $n \times n$ matrices.

1. The spaces $L^{1}(X), L^{\infty}(X)$ and $M_{b d}(X)$. We shall begin by giving a result which applies to a fairly general type of subspace of $M_{b d}(X)$.

1.1 THEOREM. Let $F$ be a left [right] translation-invariant vector subspace of $M_{b d}(X)$, and suppose that the following condition is fulfilled-

There exists a representing set $X^{\prime}=\{\xi\}$ for $X$ such that,

for each $\xi \in X^{\prime}, \hat{F}(\xi)=\{\hat{f}(\xi): f \in F\}$ is either $\{0\}$,

or one-dimensional, or the whole of $E_{\xi}$.

Let $T$ be an endomorphism of $F$ such that, for each $f \in F, T f$ is the $\sigma\left(M_{b d}, C_{b d}\right)$-limit of finite linear combinations of left-[right] translates of $f$. Then to each $\xi \in X^{\prime}$ corresponds $c(\xi) \in E_{\xi}$ such that

$$
\widehat{T f}(\xi)=c(\xi) \hat{f}(\xi) \quad[\hat{f}(\xi) c(\xi)]
$$

holds for all $f \in F$ and all $\xi \in X^{\prime}$.

Proof. A trivial computation shows that

$$
\widehat{\tau_{a} f}(\xi)=\xi(a) \hat{f}(\xi) \text { and } \widehat{\rho_{a} f}(\xi)=\widehat{f}(\xi) \xi(a)
$$

for $a \in X, \xi \in X^{\prime}$ and $f \in M_{b d}(X)$. As a consequence, $\widehat{T f}(\xi)$ belongs to to the left [right] ideal in $E_{\xi}$ generated by $\hat{f}(\xi)$. (We are here using the fact that all vector subspaces of $E_{\xi}$ are closed.) In particular, $\hat{f}(\xi)=0$ entails $\widehat{T f}(\xi)=0$.

Take a fixed $\xi$. If $\hat{F}(\xi)$ is either $\{0\}$ or one-dimensional, the existence of $c(\xi)$ for which (1) holds for all $f \in F$ is evident. Otherwies, $\hat{F}(\xi)=$ $E_{\xi}$. So, given $A \in E_{\xi}$, there exists $f \in F$ satisfying $\hat{f}(\xi)=A$. However $f \in F$ be chosen in this way, $\widehat{T f}(\xi)$ depends only upon $A$ and not upon $f$. Define $J(A)=\widehat{T f}(\xi)$. Evidently, $J$ is a linear map of $E_{\xi}$ into itself. Moreover, $J(A)$ belongs to the left [right] ideal generated by $A=\hat{f}(\xi)$. To complete the proof one has only to appeal to the lemma in 0.4 .

1.2 Corollary. If $F, X$ and $T$ are as in Theorem 1.1, then

$$
T\left(\rho_{a} f\right)=\rho_{a}(T f) \quad\left[T\left(\tau_{a} f\right)=\tau_{a}(T f)\right]
$$


and

$$
T(f * g)=T f * g \quad[T(f * g)=f * T g]
$$

whenever $f, g$ and $f * g \in F$ and $a \in X$.

1.3 Remark. If $X$ is compact, and if we take for $X^{\prime}$ a complete set of inequivalent, irreducible, continuous, unitary representations of $X$ (see 0.2 ), then condition $(*)$ is fulfilled if $F=C(X)$ (here identical with $C_{b d}(X), C_{0}(X)$ and $\left.C_{c}(X)\right)$, or $L^{p}(X)(1 \leqq p \leqq \infty)$, or $M_{b d}(X)$. If $X$ is abelian, and if we take $X^{\prime}=\hat{X}$ (see 0.2 ), condition $(*)$ is fulfilled when $F=M_{b d}(X), L^{1}(X)$, or $C_{c}(X)$.

1.4 REMARK. When $X$ is abelian, we may take $X^{\prime}=\hat{X}$, and identify $\hat{f}$ with the ordinary scalar-valued Fourier transform of $f$. Then (1) says that $T$, or the associated scalar-valued function $c$ on $\hat{X}$, is a Fourier factor or multiplier for the set $F$ of functions or measures in question. As we shall see in the next corollary this permits us to characterise $T$ still more closely. In any event it is noteworthy that (1) is enough to show that $T$ has a closed graph whenever $F$ is endowed with a vector space topology stronger than that induced on it by $\sigma\left(M_{b d}, C_{b d}\right)$. If $F$, so topologised, is such that the Closed Graph Theorem is valid for endomorphisms of $F$ (for example, if $F$ is a Fréchet space or a strict inductive limit of Fréchet spaces), it follows that $T$ is automatically continuous on $F$. This is therefore the case if $F$ is $L^{1}(X)$ or $M_{b d}(X)$, or if $X$ is compact and $F=C(X)$ or $L^{p}(X)$ with $1 \leqq p \leqq \infty$.

1.5 THEOREM. Suppose that $F=L^{1}(X)$ or $M_{b d}(X)$ and that condition (*) of Theorem 1.1 is fulfilled. If $T$ is an endomorphism of $F$ with the property that, for each $f \in F, T f$ is the $\sigma\left(M_{b d}, C_{b d}\right)$-limit of finite linear combinations of left [right] translates of $f$, then there exists $\mu \in M_{b d}(X)$ such that

$$
T f=\mu * f \quad\lfloor f * \mu]
$$

for all $f \in F$.

Proof. In view of Remark 1.4 we have only to verify that any continuous endomorphism $T$ of $F$ which satisfies (3) for arbitrary $f, g \in F$ has the stated from (4). When $X$ is abelian and $X^{\prime}$ is taken to be $X$, this result is well-known. Since the argument is rapid, we reproduce it here. ${ }^{1}$

If $F=M_{b d}(X)$, one has only to take $f=\varepsilon$ (the Dirac measure

1 From this point on we shall write out the proofs for the "left-handed" case only, leaving the reader to make the minor modifications necessary in the alternative case. 
placed at the neutral element $e$ of $X$ ) in (3) and then replace $g$ by $f$.

Suppose that $F=L^{1}(X)$. Take a directed family $\left(f_{i}\right)$ in $L^{1}(X)$ such that $f_{i} \geqq 0, \int f_{i} d x=1$, and such that the support of $f_{i}$ ultimately lies within any preassigned neighbourhood of $e$. Then $\lim \left(f_{i} * g\right)=g$ in $L^{1}$ and so, $T$ being continuous, (3) yields

$$
T g=\lim \left(h_{i} * g\right) \quad \text { in } L^{1},
$$

where $h_{i}=T f_{i} \in L^{1}$. Now the $h_{i}$ are bounded in $L^{1}$, again by continuity of $T$. The directed family $\left(h_{i}\right)$ therefore admits in $M_{b d}$ a $\sigma\left(M_{b d}, C_{0}\right)$ limiting point, say $\mu$. If then $g$ belongs to $C_{0}, \mu * g$ is a limiting point of the family $\left(h_{i} * g\right)$ for the topology of locally uniform convergence on $X$. To see this one need only observe that the $h_{i}$ are bounded in $L^{1}$, that the left translates $\tau_{a} g$ corresponding to any compact set of $a$ 's form a compact subset of $C_{0}$, and use Ascoli's Theorem. Consequently, if $g \in C_{0} \cap L^{1}, T g$ and $\mu * g$ must represent the same element of $L^{1}$. In other words, $T g=\mu * g$ for $g \in C_{0} \cap L^{1}$. Now both sides of this expression are continuous in $g \in L^{1}$, and $C_{0} \cap L^{1}$ is dense in $L^{1}$. So one obtains (4) (with $g$ in place of $f$ ).

1.6 Theorem. Suppose that the hypotheses of Theorem 1.1 are fulfilled when $F=L^{1}(X)$. Let $T$ be an endomorphism of $L^{\infty}(X)$ which is continuous for $\sigma\left(L^{\infty}, L^{1}\right)$ and such that, for each $f \in L^{\infty}(X), T f$ is the $\sigma\left(L^{\infty}, L^{1}\right)$-limit of finite linear combinations of left [right] translates of $f$. Then there exists $\mu \in M_{b d}(X)$ such that (4) holds for $f \in L^{\infty}(X)$.

Proof. The dual of $L^{\infty}$ relative to $\sigma\left(L^{\infty}, L^{1}\right)$ is $L^{1}$. So, if $S$ is the adjoint of $T, S$ is an endomorphism of $L^{1}$ which is continuous for $\sigma\left(L^{1}, L^{\infty}\right)$ and hence (by the Closed Graph Theorem) for the normed topology of $L^{1}$

We aim to show that if $g \in L^{1}$, then $S g$ is the limit in $L^{1}$ of finite linear combinations of left translates of $g$. In fact, if $f \in L^{\infty}$, and if $\int g\left(\tau_{a} f\right) d x=0$ for all $a \in X$, then $\int g(T f) d x=0$. This signifies that if $\int\left(\tau_{a-1} g\right) f d x=0$ for all $a \in X$, then $\int(S g) f d x=0$. The HahnBanach Theorem therefore leads to the stated conclusion.

To $S$ we may apply Corollary 1.5: there existe $\lambda \in M_{b d}(X)$ such that $S g=\lambda * g$ for all $g \in L^{1}$. So for arbitrary $f \in L^{\infty}$ one has

$$
\int g(T f) d x=\int(S g) f d x=\int(\lambda * g) f d x=\int g(\check{\lambda} * f) d x,
$$

the last step by use of the Lebesgue-Fubini Theorem and $\check{\chi}$ denoting the image of $\lambda$ under the map $x \rightarrow x^{-1}$ of $X$ onto itself. Putting $\mu=\check{\lambda}$, we see that $T f=\mu * f$ and the proof is complete. 
1.7 REMARK. It is natural to speculate whether the result of Corollary 1.6 holds without any a priori restriction of continuity on $T$. For compact groups, see Theorem 2.3 below.

1.8 Modifications to Theorems 1.1 and 1.5. Of the numerous possible modifications we mention only two.

In the first place one may in each of these theorems replace the topology $\sigma\left(M_{b d}, C_{b d}\right)$ by a topology $\sigma\left(M_{b d}, P\right)$, where $P$ is a subset of $C_{b d}(X)$ which contains all the coordinates of all the representations $\xi \in X^{\prime}$. (By a coordinate of $\xi$ we shall understand a function of the form $x \rightarrow\left\langle\xi(x) v, v^{\prime}\right\rangle$, where $v$ is an arbitrary vector in $V_{\xi}$ and $v^{\prime}$ an arbitrary linear form on $V_{\xi}$. If $\xi$ is given in matricial form, one may interpret a coordinate of $\xi$ to mean a function $x \rightarrow \xi_{i j}(x)$, where $\xi_{i j}(x)$ is the $(i, j)$ th entry in the matrix $\xi(x)$.)

In the second place one may vary the content of either theorem by considering, in place of endomorphisms of $F=L^{1}(X)$, a linear map $T$ of $L^{1}(X)$ into $M_{b d}(X)$. Regarding $L^{1}$ as injected into $M_{b d}$, one may consider those $T$ which satisfy the same approximation condition as before (possibly relacing $\sigma\left(M_{b d}, C_{b d}\right)$ by $\left.\sigma\left(M_{b d}, P\right)\right)$. The conclusions would be as before: $T$ would satisfy conditions (1), (2) and (3) and would admit a representation (4).

An especially significant choice of $P$ is possible in case $X$ is a compact Lie group. It is known $([5], \S 40)$ that each coordinate of each continuous, finite-dimensional representation of $X$ is analytic, a fortiori belongs to $C^{\infty}(X)$. One may therefore take $P=C^{\infty}(X)$. Then the topology $\sigma(M, P)$ is that induced on $M$ by the notion of distributional convergence. Thus our requirement on $T$ is that $T f$ be the distributional limit of finite linear combinations of left [right] translates of $f$.

2. The case in which $X$ is compact. In this case, as we have noted in Remark 1.3, if $F$ is any one of $C(X), L^{p}(X)(1 \leqq p \leqq \infty)$ or $M(X)$ (here identical with $M_{b d}(X)$ ), and if $T$ is any endomorphism of $F$ such that $T f$ is always the $\sigma(M, C)$-limit of finite linear combinations of left [right] translates of $f$, then (1), (2) and (3) are true for arbitrary $f, g \in F$. In particular, in all these cases the Closed Graph Theorem shows that $T$ is necessarily continuous for the respective normed topologies. Yet it still remains to consider the formula (4) for cases other then $L^{1}(X)$ and $M(X)$, which have been dealt with in Theorem 1.5. Theorem 1.6 gives a partial answer in the case of $L^{\infty}(X)$, provided continuity of $T$ for $\sigma\left(L^{\infty}, L^{1}\right)$ is assumed. In case $F=L^{p}(X)$ with $1<p<\infty$, the formula (4) will not in general be valid; the simplest counter-example is perhaps that when $p=2$ and $X$ is the circle group. The problem of Fourier multipliers for $L^{p}$ is a famous one; for a detailed study of the case in which $X=R^{n}$, see [3]. 
We proceed to examine the cases $F=C(X)$ and $F=L^{\infty}(X)$ when $X$ is compact.

2.1 THEOREM. Let $X$ be a compact group, $T$ an endomorphism of $C(X)$ such that, for each $f \in C(X)$, Tf is the $\sigma(M, C)$-limit of finite linear combinations of left [right] translates of $f$. Then there exists a measure $\mu \in M(X)\left(=M_{b d}(X)\right)$ such that (4) holds for all $f \in C(X)$.

Proof. We know that $T$ is continuous and satisfies (2). Let $S$ be the adjoint of $T . S$ is an endomorphism of $M$ which is continuous for the normed topology and for the weak topology $\sigma(M, C)$. By (2) and the defining property of $S$ we obtain

$$
\begin{aligned}
\int f d\left(S \rho_{a} \lambda\right) & =\int(T f) d\left(\rho_{a} \lambda\right)=\int\left(\rho_{a^{-1}} T f\right) d \lambda=\int\left(T \rho_{a^{-1}} f\right) d \lambda \\
& =\int\left(\rho_{a^{-1}} f\right) d(S \lambda)=\int f d\left(\rho_{a} S \lambda\right)
\end{aligned}
$$

remembering that $X$ is unimodular $(\Delta \equiv 1)$. This shows that $S$ commutes with right translations.

Now if $\alpha$ and $\beta$ belong to $M(X)$ one may write

$$
\alpha * \beta=\int\left(\rho_{x} \alpha\right) d \beta(x),
$$

the integrand being a continuous function from $X$ into $M(X)$ when the latter is endowed with the vague topology $\sigma(M, C)$. Since $S$ is continuous for this topology it follows that

$$
S(\alpha * \beta)=\int\left(S \rho_{x} \alpha\right) d \beta(x)=\int\left(\rho_{x} S \alpha\right) d \beta(x)=S \alpha * \beta .
$$

Taking $\alpha=\varepsilon$ we obtain

$$
S \beta=\pi * \beta
$$

with $\pi=S \varepsilon \in M(X)$. Returning to the defining formula for $S$ in terms of $T$, this is seen to imply that $T f=\mu * f$ with $\mu=\check{\pi} \in M(X)$.

2.2 In view of the contents of Remark 1.8 , if $X$ is a compact Lie group it is sufficient in Theorem 2.1 to assume that $T f$ is the distributional limit of finite linear combinations of left [right] translates of $f$.

It is now possible to give a refinement of Theorem 1.6 for the case where $X$ is compact.

2.3 TheOREM. Let $X$ be compact and let $T$ be an endomorphism of $L^{\infty}(X)$ such that, for each $f \in L^{\infty}(X)$, Tf is the $\sigma(M, C)$-limit of 
finite linear combinations of left [right] translates of $f$. Then there exists $\mu \in M(X)$ such that (4) is true for all continuous $f$.

Proof. Take any $u \in L^{1}$ and consider the endomorphism $T_{u}$ of $C$ defined by $T_{u} f=u * T f$. We claim that for each continuous $f, T_{u} f$ is the $\sigma(M, C)$-limit of finite linear combinations of functions $\tau_{a} f$. To justify this we must show (Hahn-Banach theorem) that if $g \in C$ satisfies $\int\left(\tau_{a} f\right) g d x=0$ for all $a \in X$, then $\int\left(T_{u} f\right) g d x=0$. Now

$$
\begin{aligned}
\int\left(T_{u} f\right) g d x & =\int(u * T f) g d x=\int g(x) d x \int u(y) T f\left(y^{-1} x\right) d y \\
& =\int u(y) d y \int T f\left(y^{-1} x\right) g(x) d x=\int u(y) d y \int T f\left(x^{\prime}\right) g\left(y x^{\prime}\right) d x^{\prime}
\end{aligned}
$$

The main hypothesis on $T$ ensures that $\int T f\left(x^{\prime}\right) g\left(y x^{\prime}\right) d x^{\prime}=0$ whenever $g \in C$ and $\int\left(\tau_{a} f\right) g d x=0$ for all $a \in X$. So, under the same hypotheses, $\int\left(T_{u} f\right) g d x=0$, which is what we had to show.

We can at this point apply Theorem 2.1 to $T_{u}$ and conclude the existence of $\mu_{u} \in M$ such that

$$
T_{u} f=\mu_{u} * f
$$

for all $f \in C$ and all $u \in L^{1}$.

Consider the mapping $U: u \rightarrow \mu_{u}$ of $L^{1}$ into $M$. It is evident that $\mu_{u}$ is uniquely determined by $u$, so that $U$ is well-defined, and also that $U$ is linear. An easy application of the Closed Graph Theorem shows that $U$ is continuous for the normed topologies. Thus there is a number $c$ such that $\int d\left|\mu_{u}\right| \leqq c \cdot \int|u| d x$. Take $a$ directed family $\left(u_{i}\right)$ in $L^{1}$ with the same properties as the family $\left(f_{i}\right)$ in the proof of Theorem 1.5. The corresponding $\mu_{u_{i}}=\mu_{i}$ and then bounded in norm and so have a $\sigma(M, C)$-limiting point $\mu$. On the other hand, $T_{u_{\imath}} f=$ $u_{i} * T f$ converges weakly in $L^{\infty}$ to $T f$, whilst the $\mu_{i} * f$ will have $\mu * f$ as a limiting point for the topology of uniform convergence on $X$. By comparison we see that the classes $T f$ and $\mu * f$ are identical, and this for each $f \in C$. The proof is complete.

2.3 Remark. Given that $T$ is continuous for $\sigma\left(L^{\infty}, L^{1}\right)$, it is easy to conclude that (4) continues to hold for all $f \in L^{\infty}$. Thus the result of Theorem 1.6 is recovered for $X$ compact.

3. Continuous functions on an abelian group. Theorem 2.1 deals effectively with our problem for the space of continuous functions on any compact group. We turn next to the case of non-compact groups 
$X$. Here an effective solution is forthcoming only when $X$ is abelian, in which case we shall follow normal usage and write $X$ additively.

One minor variant will be introduced, namely that of restricting attention to the left translates $\tau_{a} f$ corresponding to those a belonging to a pre-assigned closed subset $Y$ of $X$.

We use throughout the "natural" topology of $C=C(X)$, which is that defined by the seminorms

$$
p_{K}(f)=\operatorname{Sup}\{|f(x)|: x \in K\},
$$

$K$ running over all (or over a base for all) the compact subsets of $X$.

3.1 TheOREM. Let $X$ be abelian, and let $T$ be an endomorphism of $C(X)$ which is continuous and such that, for some pre-assigned closed subset $Y$ of $X$ and each $f \in C(X), T f$ is the $\sigma\left(M, C_{c}\right)$-limit of finite linear combinations of translates $\tau_{a} f$ with $a \in Y$. (If $X$ is a finite product of lines and circles, it suffices to replace $\sigma\left(M, C_{c}\right)$ convergence by distributional convergence.) Then there a exists measure $\mu$ on $X$ with support lying in a set $Y \cap K$, where $K$ is compact, and such that (4) holds for all $f \in C(X)$.

Proof. Introduce the dual group $\hat{X}$ and denote its members by $\xi$. Our main hypothesis on $T$ ensures that $T \xi=c(\xi) \xi$, where the scalar $c(\xi)$ depends upon $\xi$. Plainly, $c(\xi)=T \xi(0), 0$ denoting the neutral element of $X$.

Let $\left(\alpha_{i}\right)$ be any family of scalars, zero for all but a finite set of indices $i$. We have then

$$
\Sigma \alpha_{i} c\left(\xi_{i}\right)=\Sigma \alpha_{i} \cdot T \xi_{i}(0)=T\left(\Sigma \alpha_{i} \xi_{i}\right)(0) .
$$

Since $T$ is continuous on $C$, it follows that there exists a compact subset $K$ of $X$ and a number $M$ such that

$$
|T f(0)| \leqq M \cdot p_{K}(f)
$$

for all $f \in C$. So in particular we discover that

$$
\left|\Sigma \alpha_{i} c\left(\xi_{i}\right)\right| \leqq M \cdot p_{K}\left(\Sigma \alpha_{i} \xi_{i}\right) \text {. }
$$

By applying the Hahn-Banach Theorem to the space $C(K)$ of continuous functions on $K$, endowed with the topology of uniform convergence on $K$, it is seen to follow that there exists a Radon measure $\mu$ on $X$ with support contained in $K$ such that

$$
c(\xi)=\int \xi(y) d \mu(y)
$$

for all $\xi$. For convenience we replace $\mu$ by $\check{\mu}$ and $K$ by $-K$, which 
enables us to rewrite this last relation in the form

$$
c(\xi)=\int \xi(-y) d \mu(y) \text {. }
$$

The linearity of $T$ now shows that

$$
T f(x)=\int f(x-y) d \mu(y)=\mu * f(x)
$$

for all $f \in C$ which are finite linear combinations of characters $\xi \in \hat{X}$. These finite linear combinations are dense in $C$, and the continuity of $T$ shows therefore that $T f=\mu * f$ holds for all $f \in C$.

It remains to show that the support of $\mu$ is contained in $Y$. Let $V$ be any compact neighbourhood of 0 in $X$ and let $k \in C_{c}(X)$ have its support inside $V$. (If $X$ is a finite product of lines and circles we choose $k$ in $C_{c}^{\infty}(X)$.) Then our hypothesis concerning the approximation of $T f$ entails that $(T f) * k=\mu * k * f$ is the limit locally uniformly of translates $\tau_{a} f$ with $a \in Y+V$. So if $\lambda$ is any measure with a compact support satisfying $\int f(x-a) d \lambda(x)=0$ for $a \in Y+V$, then $\int(\mu * k * f) d \lambda=$ 0 . This is true for any $f \in C$. Taking $f$ so that $f(-a)=0$ for $a \in Y+V$ and then $\lambda=\varepsilon$, we conclude that $\mu * k * f(0)=0$, i.e. that

$$
\int f(-x) d(k * \mu)(x)=0 \text {. }
$$

In other words,

$$
\int g d(k * \mu)=0
$$

for any $g \in C$ which vanishes on $Y+V$. This entails that $k * \mu$ has its support within $Y+V$. By varying $k$ suitably, making $k d x$ converge vaguely to $\varepsilon$, it may be concluded that the support of $\mu$ lies in $Y+V$. Since $V$ is an arbitary compact neighbourhood of zero, the support of $\mu$ must lie within $Y$. This completes the proof.

3.2 ExAmple. If we suppose that $Y$ is a discrete subset of $X$, the measure $\mu$ has a finite support and we conclude that $T$ must have the simple form:

$$
T f(x)=\sum_{i=1}^{n} c_{i} f\left(x-a_{i}\right),
$$

where the $c_{i}$ are scalars and the $a_{i}$ are points of $Y$.

3.3 Remarks. I do not know whether the result stated in Theorem 3.1 is valid for non-abelion, non-compact $X$. Nor do I know whether 
the a priori hypothesis of continuity of $T$ can be removed.

4. The case $X=R^{n}, F=C_{c}^{\infty}$ or $\mathscr{D}^{\prime}$. We combine into one theorem the results applying to these two cases.

4.1 TheOREM. Let $X=R^{n}$, and let $T$ be a continuous endomorphism of $\mathscr{D}^{\prime}\left[\right.$ resp. of $C_{c}^{\infty}$ ] with the property that for each $f \in \mathscr{D}^{\prime}$ [resp. $f \in C_{c}^{\infty}$ ] Tf is the limit in $\mathscr{D}^{\prime}$ [resp. in $C_{c}^{\infty}$ ] of translates of $f$. Then there exists a distribution $\mu$ with a compact support such that (4) holds for all $f \in \mathscr{D}^{\prime}$ [resp. $f \in C_{c}^{\infty}$ ].

Proof. (i) Take the case of $\mathscr{D}^{\prime}$ first. As before, the behaviour of $T$ acting on continuous characters is prescribed by the rule

$$
T\left(e^{-2 \pi i \xi \cdot x}\right)=c(\xi) e^{-2 \pi i \xi \cdot x} ;
$$

here $\xi$ and $x$ are points of $R^{n}$ and $\xi \cdot x$ denotes the usual Euclidean scalar product of these two vectors.

If $T^{\prime}$ is the adjoint of $T$, then $T^{\prime}$ is a continuous endomorphism of $C_{c}^{\infty}$ and it appears at once that

$$
\widehat{T^{\prime} g}(\xi)=c(\xi) \hat{g}(\xi) \text {. }
$$

From this it appears that $T^{\prime}$ commutes with translations, so that the same must be true of $T$. Hence, by a theorem of Schwartz ([6], p. 18 , Théorème $\mathrm{X}$ and p. 19, Remarque), $T f=\mu * f$ for some distribution $\mu$ with a compact support.

(ii) Now consider the case of $C_{c}^{\infty}$. The adjoint $T^{\prime}$ of $T$ is now an endomorphism of $\mathscr{D}^{\prime}$ which is continuous and which has the property stated in the theorem. It follows from (i) that $T^{\prime} g=\lambda * g$ for all $g \in \mathscr{D}^{\prime}$, where $\lambda$ is a suitable distribution with a compact support. This leads to $T f=\mu * f$, where $\mu=\check{\lambda}$. The proof is complete.

4.2 Remark. There is a corresponding result if $X$ is a finite product of circle groups, say $K^{n}$, or if $X$ is a product $R^{n} \times K^{m}$.

4.3 Remark. Once again, I do not know whethor the a priori assumption of continuity of $T$ can be removed; cf. Remark 3.3.

5. Some applications. The existence of a representation formula of the type (4) for certain linear maps $T$ has some interesting consequences, on a sample of which we comment.

5.1 Theorem. Let $\left(\mu_{i}\right)$ be a directed family in $M_{b d}(X)$ and suppose that $\left(\mu_{i} * f\right)$ is $\sigma\left(M_{b d}, C_{b d}\right)$-convergent for each $f \in L^{1}(X)$. Then there 
exists $\mu \in M_{b d}(X)$ such that

$$
\lim \left(\mu_{i} * f\right)=\mu * f
$$

for each $f \in L^{1}(X)$.

Proof. Let $\lambda_{f}$ denote the said limit of $\mu_{i} * f$. It is evident that $T: f \rightarrow \lambda_{f}$ is a linear map of $L^{1}$ into $M_{b d}$. Moreover $T f$ is the $\sigma\left(M_{b d}, C_{b d}\right)$ limit of finite linear combinations of left translates $\tau_{a} f$, since the same is easily seen to be the case of $\mu_{i} * f$ for each $i$. So, by what has been said in 1.8, $T f=\mu * f$ for some $\mu \in M_{b d}$, as alleged.

5.2 REMARKs. It is easily seen that $T$ commutes with right translations. In those cases where one can affirm that $T$ is continuous (which includes the case in which $\left(\mu_{i}\right)$ is a denumerable sequence, and that in which $\left(\mu_{i}\right)$ is norm-bounded in $\left.M_{b d}(X)\right)$, the same result would follow when (4) is known for the continuous linear maps of $L^{1}(X)$ into $M_{b d}(X)$ which commute with right translations.

If $X$ is compact, the $\sigma(M, C)$-limit of $\mu_{i} * f$ exists in either of the following two cases-

(i) the $\mu_{i}$ are bounded in norm and

$$
\lim _{i} \int\left(\mu_{i} * f\right) g d x
$$

exists finitely for each $g \in C(X)$;

(ii) $\left(\mu_{i}\right)$ is a denumerable sequence and the limit (5) exists finitely for each $g \in C(X)$.

Notice also that

$$
\int\left(u_{i} * f\right) g d x=\int h d \mu_{i}
$$

where

$$
\begin{aligned}
h(x) & =\int f\left(x^{-1}\right) g(y) d y=g * \check{f}(x) \\
& =\int f(y) g(x y) d y=\int f(y) g\left(y^{-1} x^{-1}\right)=f * \check{g}\left(x^{-1}\right)
\end{aligned}
$$

Thus the limit (5) exists finitely for $f \in L^{1}(X)$ and $g \in C(X)$ if and only if $\left(\mu_{i}\right)$ is Cauchy for the topology $\sigma\left(M, C * L^{1}\right)$ (or, what is equivalent, for the topology $\left.\sigma\left(M, L^{1} * C\right)\right)$.

If $X$ is a compact Lie group one may in Theorem 5.1 assume merely that $\left(\mu_{i} * f\right)$ is distributionally convergent to some measure for each $f \in L^{1}(X)$.

5.3 By starting from Theorem 4.1 one may infer the following 
analogue of Theorem 5.1-if $\left(\mu_{i}\right)$ is a directed family of measures in $M_{c}\left(R^{n}\right)$, and if $\left(\mu_{i} * f\right)$ is distributionally convergent to a function in $C\left(R^{n}\right)$ for each $f \in C\left(R^{n}\right)$, then there exists $\mu \in M_{c}\left(R^{n}\right)$ such that $\mu_{i} * f \rightarrow \mu * f$ distributionally for each $f \in C\left(R^{n}\right)$.

5.4 From 5.2 and 5.3 it follows that if $X$ is $R^{n}$ or a compact Lie group, and if $\mu$ is a distribution on $X$ with a compact support such that $\mu * f$ is a continuous function for each continuous function $f$, then $\mu$ is necessarily a measure. Compare [6], p. 48, Théorème XX.

\section{Some related problems.}

6.1 In several cases already discussed, $F$ is an algebra under convolution; such is the case if $F=L^{1}(X)$ or $M_{b d}(X)$, or if $X$ is compat and $F=C(X)$ or $F=L^{p}(X)$ for $1 \leqq p \leqq \infty$. If at the same time $F$ is a topological algebra (which is so in the instances just cited), to say that $T f$ is the limit in $F$ of finite linear combinations of left translates of $f$ is frequently equivalent to saying that $T f$ belongs to the closed left ideal in $F$ generated by $f$.

So one is led naturally to the following question for topological algebras $F$. What vector-space endomorphisms $T$ of $F$ (which may or may not be assumed a priori to be continuous) possess the property that, for each $f \in F, T f$ belongs to the closed left ideal generated by $f$ ? It comes to the same thing to demand that $T$ leaves stable all closed left ideals in $F$.

In those cases in which $F$ is algebraically isomorphic with some algebra (under pointwise operations) of functions defined on some set, and convergence in $F$ entails pointwise convergence of the image functions under the isomorphism, the problem can be solved with the aid of the following lemma.

Lemma. Let $X$ be a set, $R$ a ring, and $R^{x}$ the set of $R$-valued functions on $X$ considered as a left $R$-module. Suppose that $F$ is a submodule of $R^{x}$ with the property that for each $x \in X$ there exists $f_{x} \in F$ for which $f_{x}(x)$ is a right identity in $R$, and that $T$ is a $R$ homomorphism of $F$ into $R^{x}$ with the property that $f \in F, x \in X$ and $f(x)=0$ together entail that $T f(x)=0$. Then there exists $c \in R^{x}$ such that

$$
T f(x)=f(x) c(x)
$$

for all $f \in F$ and $x \in X$.

Proof. Given $f \in F$ and $x \in X$ consider $g \in F$ defined by

$$
g=f-f(x) f_{x} \text {. }
$$


Evidently, $g(x)=0$. This signifies that

$$
T f(x)=f(x) T f_{x}(x),
$$

whence the assertion on taking $c(x)=T f_{x}(x)$.

The following result is now deducible.

THEOREM. Let $A$ be an algebra over a topological field $K, A$ being endowed with some topology. Suppose there exists a set $X$ and an algebraic isomorphism $f \rightarrow \hat{f}$ of $A$ into $K^{x}$ such that (i) for each $x \in X$ there exists $f_{x} \in A$ for which $\hat{f}_{x}(x) \neq 0$, and (ii) $\lim f_{i}=f$ in $A$ entails that $\lim \hat{f}_{i}=\hat{f}$ pointwise on $X$. Finally suppose that $T$ is a vectorspace endomorphism of $A$ with the property that, for each $f \in A, T f$ belongs to the closure of the ring-ideal in A generated by $f$, i.e. $T f=$ $\lim g_{i} f$ for a suitable directed family $\left(g_{i}\right)$ of elements of $A$. Then there exists a K-valued function $c$ on $X$ such that

$$
\widehat{T f}(x)=c(x) \hat{f}(x)
$$

for all $f \in A$ and $x \in X$. In particular $T$ is a multiplier of $A$, i.e.

$$
T(f g)=(T f) g=f(T g)
$$

for $f, g \in A$.

Proof. Let $F \subset K^{x}$ be the image of $A$ under the isomorphism $f \rightarrow \hat{f}$ and define $\hat{T}$ as a $K$-linear map of $F$ into $K^{x}$ by the formula

$$
\hat{T} \hat{f}=(T f)^{\wedge} \text {. }
$$

If $f \in F, x \in X$ and $\hat{f}(x)=0$ then one has

$$
\hat{T} \hat{f}(x)=\widehat{T f}(x)=\lim \widehat{g_{i} f}(x)=\lim \hat{g}_{i}(x) \hat{f}(x)=\lim 0=0 .
$$

The hypotheses of the preceding lemma are fulfilled if we take $R=K$ and $\hat{T}$ in place of $T$, whence the theorem.

REMaRKs. The best-known case in which the theorem is applicable is that in which $A$ is a semisimple commutative Banach algebra, $X$ being taken to be the "character space" of $A$ (in one-to-one correspondence with the space of regular maximal ideals in $A$ ) and $f \rightarrow \hat{f}$ the Gelfand transform. In this case $X$ carries a natural topology making it into a separated locally compact space in such a way that each $\hat{f}$ is continuous and vanishes at infinity on $X$. (If $A$ has an identity $X$ is compact.) In this case (6) shows at once that $c$ is continuous on $X$. At the same time (7) shows that $T$ has a closed graph and is therefore continuous on $A$. Finally, one can use an argument of Wang ([4], Theorem 3.1) to deduce that $c$ is necessarily bounded on $X$. 
In most cases, $c$ has to be further restricted in order that the product function $c \hat{f}$ shall be the transform of some element of $A$ for each $f \in A$.

For commutative, semisimple, Banach algebras a closely related result is established by Birtel [1], Theorem 3 .

6.2 Another natural extension to the problem we have been considering is as follows. Suppose $X$ is an arbitrary set and $Q=\{q\}$ a set of self-maps of $X$. Consider a topological vector space $F$ of functions on $X$ which is invariant under $Q$ in the sense that $f_{q}=f \circ q \in F$ whenever $f \in F$ and $q \in Q$. One may then ask: What are the endomorphisms $T$ of $F$ with the property that, for each $f \in F, T f$ is the limit in $F$ of finite linear combinations of functions $f_{q}(q \in Q)$ ? One may or may not assume a priori that $T$ is continuous on $F$. Consideration of a few simple examples would indicate that the problem, thus stated, is too general for there to be any hope of a simple representation formula similar to (4) for such endomorphisms $T$.

A simple type of example arises of one takes $X=R^{n}, F=C\left(R^{n}\right)$ and for $Q$ the set of self-maps of $X$ of the type

$$
x \rightarrow A x+b, \quad A \in \mathscr{A}, b \in B,
$$

where $\mathscr{A}$ is a set of vector space endomorphisms of $R^{n}$ and $B$ a subset of $R^{n}$. If $\mathscr{A}$ and $B$ are reasonably large, it will appear that our hypothesis concerning $T$ is too weak to be useful, simply because for all but very restricted functions $f$ the set $M_{f}$ of finite linear combinations of the $f_{q}$ are already dense in $F$.

Thus it is known (see [7]) that if $B=R^{n}$ and $\mathscr{A}$ contains all homothetic maps $x \rightarrow \lambda x$ with $\lambda>0$ (or merely those corresponding to a set of $\lambda$ having 0 as a limiting point), then $M_{f}=C\left(R^{n}\right)$ unless $f$ is a distributional solution of some linear partial differential equation with constant coefficients; if $n=1, M_{f}$ will coincide with $C\left(R^{n}\right)$ unless $f$ is a polynomial. Whatever the value of $n$, if moreover $\mathscr{A}$ contains all rotations of $R^{n}$, then $M_{f}=C\left(R^{n}\right)$ unless $f$ is polyharmonic (i.e., is distributionally the solution of an iterated Laplace equation $\Delta^{m} f=0$ ).

In view of these results, if $n$ is taken to be 1 , then $T$ will satisfy our hypothesis, relative the case in which $Q$ consists of all maps $x \rightarrow$ $a x+b$ ( $a, b$ real), provided simply that $T$ transforms the monomial $x^{k}$ into a polynomial of degree at most $k$, and this for $k=0,1,2, \cdots$. Now it is easy to construct a great variety of such endomorphisms $T$ which are continuous. One has simply to define $T$ by

$$
T f(x)=\int f d \mu_{x}
$$

where $\mu_{x}$ is a measure of the type 


$$
\mu_{x}=\sum_{k=0}^{\infty} P_{k}(x) \lambda_{k}
$$

$P_{k}$ being a polynomial of degree at most $k$ such that, if

$$
A_{k}(r)=\sup _{|x| \leqq r}\left|P_{k}(x)\right| \text {, }
$$

one has

$$
\sum_{k} A_{k}(r)<+\infty
$$

for each $r>0$, whilst the $\lambda_{k}$ are measures supported by a compact set independent of $k$ and satisfying

$$
\int x^{p} d \lambda_{k}(x)=0 \text { for } 0 \leqq p<k,
$$

and

$$
\int d\left|\lambda_{k}\right| \leqq 1 \quad \text { for } k \geqq 0 \text {. }
$$

Notice that, unlike the traslational case, such an endomorphism $T$ need not commute with the operations $f \rightarrow f_{q}$.

\section{REFERENCES}

1. F. T. Birtel, Banach algebras of multipliers, Duke Math. J., 28 (1961), 203-211.

2. P. Eymard, L'algibre de Fourier d'un groupe localement compact, C. R. Acad. Sci., Paris 256 (1963), 1429-1431.

3. L. Hörmander, Estimates for translation invariant operators in $L^{p}$ spaces, Acta Mathematica, 104 (1960), 93-140.

4. Ju-Kwei Wang, Multipliers of commutative Banach algebras, Pacific J. Math., 11 (1961), 1131-1149.

5. W. Maak, Fastperiodische Funktionen, Berlin, 1950.

6. L. Schwartz, Théorie des distributions, Tome II. Act. Sci. et Ind. No. 1122, Paris 1951.

7. — Sur certains familles non-fondamentales de fonctions continues, Bull. Soc. Math. de France (1944), 1-5.

8. A. Weil, L'intégration dans les groupes topologiques et ses applications, Act. Sci. et Ind. No. 1145, Paris 1951.

INSTITUTE OF AdVANCED STUDIES,

AUSTRALIAN NATIONAL UNIVERSITY 


\section{PACIFIC JOURNAL OF MATHEMATICS}

\section{EDITORS}

Robert Osserman

Stanford University

Stanford, California

M. G. Arsove

University of Washington

Seattle 5 , Washington
J. DugundjI

University of Southern Califorma: Los Angeles 7, California

Lowell J. Paige

University of California

Los Angeles 24, California

\section{ASSOCIATE EDITORS}

E. F. BECKENBACH

B. H. NeumanN

F. WOLF

K. YosIDA

\section{SUPPORTING INSTITUTIONS}

UNIVERSITY OF BRITISH COLUMBIA

CALIFORNIA INSTITUTE OF TECHNOLOGY

UNIVERSITY OF CALIFORNIA

MONTANA STATE UNIVERSITY

UNIVERSITY OF NEVADA

NEW MEXICO STATE UNIVERSITY

OREGON STATE UNIVERSITY

UNIVERSITY OF OREGON

OSAKA UNIVERSITY

UNIVERSITY OF SOUTHERN CALIFORNIA
STANFORD UNIVERSITY

UNIVERSITY OF TOKYO

UNIVERSITY OF UTAH

WASHINGTON STATE UNIVERSITY

UNIVERSITY OF WASHINGTON

AMERICAN MATHEMATICAL SOCIETY CALIFORNIA RESEARCH CORPORATION SPACE TECHNOLOGY LABORATORIES NAVAL ORDNANCE TEST STATION 


\section{Pacific Journal of Mathematics}

\section{Vol. 14, No. 1 \\ May, 1964}

Richard Arens, Normal form for a Pfaffian .........................

Charles Vernon Coffman, Non-linear differential equations on cones in Banach

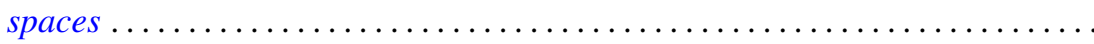

Ralph DeMarr, Order convergence in linear topological spaces ..............

Peter Larkin Duren, On the spectrum of a Toeplitz operator ................

Robert E. Edwards, Endomorphisms of function-spaces which leave stable all

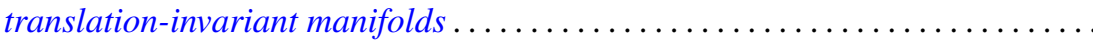

Erik Maurice Ellentuck, Infinite products of isols . . . . . . . . . . . . . . . . 49

William James Firey, Some applications of means of convex bodies . . . . . . . . 53

Haim Gaifman, Concerning measures on Boolean algebras ............. 61

Richard Carl Gilbert, Extremal spectral functions of a symmetric operator. . . . . . 75

Ronald Lewis Graham, On finite sums of reciprocals of distinct nth powers ..... 85

Hwa Suk Hahn, On the relative growth of differences of partition functions ...... 93

Isidore Isaac Hirschman, Jr., Extreme eigen values of Toeplitz forms associated

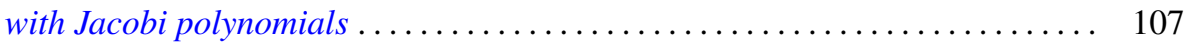

Chen-jung Hsu, Remarks on certain almost product spaces . . . . . . . . . . . 163

George Seth Innis, Jr., Some reproducing kernels for the unit disk . . . . . . . . . 177

Ronald Jacobowitz, Multiplicativity of the local Hilbert symbol . . . . . . . . . . . 187

Paul Joseph Kelly, On some mappings related to graphs ................. 191

William A. Kirk, On curvature of a metric space at a point . . . . . . . . . . . . 195

G. J. Kurowski, On the convergence of semi-discrete analytic functions . . . . . . . 199

Richard George Laatsch, Extensions of subadditive functions . . . . . . . . . . . 209

V. Marić, On some properties of solutions of $\Delta \psi+A\left(r^{2}\right) X \nabla \psi+C\left(r^{2}\right) \psi=0 \ldots 217$

William H. Mills, Polynomials with minimal value sets . . . . . . . . . . . 225

George James Minty, Jr., On the monotonicity of the gradient of a convex

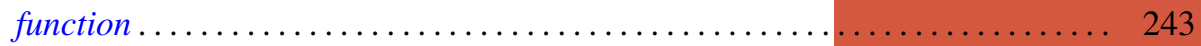

George James Minty, Jr., On the solvability of nonlinear functional equations of 'monotonic' type ................................... 249

J. B. Muskat, On the solvability of $x^{e} \equiv e(\bmod p) \ldots \ldots \ldots \ldots \ldots \ldots \ldots \ldots . \ldots \ldots$

Zeev Nehari, On an inequality of $P . R$. Bessack ................... 261

Raymond Moos Redheffer and Ernst Gabor Straus, Degenerate elliptic

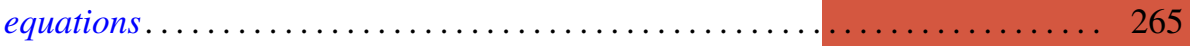

Abraham Robinson, On generalized limits and linear functionals . . . . . . . . . 269

Bernard W. Roos, On a class of singular second order differential equations with a

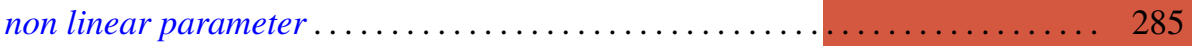

Tôru Saitô, Ordered completely regular semigroups . . . . . . . . . . . . . . . . 295

Edward Silverman, A problem of least area ....................... 309

Robert C. Sine, Spectral decomposition of a class of operators . . . . . . . . . 333

Jonathan Dean Swift, Chains and graphs of Ostrom planes . . . . . . . . . . . 353

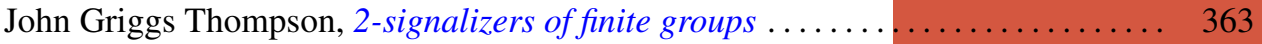

Harold Widom, On the spectrum of a Toeplitz operator . . . . . . . . . . . . . 365 